\title{
Infinito, ser ou não ser, eis a questão
}

\author{
Colaboração Matemática Aberta ${ }^{1}$
}

12 de Fevereiro de 2022

\begin{abstract}
Resumo
Apresentamos dois exemplos da Topologia que mostram a diferença entre tender ao infinito e ser infinito.
\end{abstract}

palavras-chave: infinito enumerável, interseção de conjuntos abertos, topologia

A versão mais atualizada deste artigo está disponível em https://osf.io/c5fvq/download https: //zenodo.org/record/6055109

\section{Introdução}

1. No Cálculo Diferencial e Integral, por meio de limites, já percebemos a diferença entre tender $(\rightarrow)$ e ser igual $(=)$.

2. O Princípio da Indução Matemática faz o mesmo papel de $(\rightarrow)$ em (1).

3. A seguir, apresentamos dois teoremas da Topologia que mostram $(2)$.

4. Utilizamos uma notação minimalista, sem perder informação.

${ }^{1}$ Todos os autores com suas afiliações aparecem no final deste artigo. 


\section{Notação}

5. Aberto em $\mathbb{R}$ significa conjunto aberto em $\mathbb{R}$.

6. $A, B, A_{i}:=$ conjuntos abertos em $\mathbb{R}$

7. $i \in \mathbb{N}=\{1,2,3, \ldots\}$

8. $\cap A_{i}:=$ interseção arbitrária de $A_{i}$

9. $\neg \square:=$ não necessariamente

\section{Teorema 1}

10.

$$
\bigcap_{i=1}^{n} A_{i} \equiv \text { aberto em } \mathbb{R}
$$

11. Este teorema diz que uma interseção finita de conjuntos abertos em $\mathbb{R}$ é aberta em $\mathbb{R}$.

12. O teorema (10) pode ser provado utilizando-se o Princípio da Indução Matemática (PIM) (veja os exercícios 5.iii e 11 nas pp. 132-133 de [1]).

\section{Teorema 2}

13.

$$
\bigcap_{i=1}^{\infty} A_{i} \equiv \neg \square \text { aberto em } \mathbb{R}
$$

14. Este teorema diz que uma interseção arbitrária infinita (enumerável) de conjuntos abertos não é necessariamente aberta. 


\section{Pergunta}

15. Por que a demonstração do teorema (10) por meio do PIM não produz uma contradição com o teorema (13)?

\section{Sobre os teoremas}

16. A resposta de (15) é que, enquanto o teorema (10) diz que $n \rightarrow \infty$, em (13), substitui-se $n$ por $\infty$.

\section{Considerações Finais}

17. Concluímos que tender ao infinito é diferente de ser infinito, conforme mostrado pelos dois teoremas em questão, que têm conclusões distintas.

\section{Ciência Aberta}

O arquivo latex para este artigo, juntamente com outros arquivos suplementares, estão disponíveis em [2,3]. Seja coautor(a) deste artigo, envie sua contribuição para mplobo@uft. edu.br.

\section{Consentimento}

O autor concorda com [4].

\section{Como citar este artigo?}

https://doi.org/10.31219/osf.io/c5fvq

https://zenodo.org/record/6055109 


\section{Licença}

CC-By Attribution 4.0 International [5]

\section{Referências}

[1] Warner, Steve. Topology for Beginners. GET 800, 2019.

https://books.google.com/books?id=pNAvxQEACAAJ

[2] Lobo, Matheus P. "Open Journal of Mathematics and Physics (OJMP)." OSF, 21 Apr. 2020.

https://doi.org/10.17605/osf .io/6hzyp

[3] https://zenodo.org/record/6055109

[4] Lobo, Matheus P. "Simple Guidelines for Authors: Open Journal of Mathematics and Physics." OSF Preprints, 15 Nov. 2019.

https://doi.org/10.31219/osf.io/fk836

[5] CC. Creative Commons. CC-By Attribution 4.0 International. https://creativecommons .org/licenses/by/4.0

\section{Colaboração Matemática Aberta}

Matheus Pereira Lobo ${ }^{1,2,3}$ (autor principal, mplobo@uft.edu.br) https://orcid.org/0000-0003-4554-1372

${ }^{1}$ Universidade Federal do Tocantins (Brasil)

${ }^{2}$ Universidade Federal do Norte do Tocantins (Brasil)

${ }^{3}$ Universidade Aberta (UAb, Portugal) 